\title{
Tuning of structure inversion asymmetry by the $\delta$-doping position in (001)-grown GaAs quantum wells
}

\author{
V. Lechner, ${ }^{1}$ L. E. Golub, ${ }^{2}$ P. Olbrich, ${ }^{1}$ S. Stachel, ${ }^{1}$ D. Schuh, ${ }^{1}$ W. Wegscheider, ${ }^{1, a)}$ \\ V. V. Bel'kov, ${ }^{1,2}$ and S. D. Ganichev ${ }^{1, b)}$ \\ ${ }^{1}$ Terahertz Center, University of Regensburg, 93040 Regensburg, Germany \\ ${ }^{2}$ Ioffe Physico-Technical Institute, Russian Academy of Sciences, 194021 St. Petersburg, Russia
}

(Received 6 March 2009; accepted 29 May 2009; published online 19 June 2009)

\begin{abstract}
Both structure and bulk inversion asymmetry in modulation-doped (001)-grown GaAs quantum wells were investigated employing the magnetic field induced photogalvanic effect. We demonstrate that the structure inversion asymmetry (SIA) can be accurately tailored by the delta-doping layer position. Symmetrically doped structures exhibit a substantial SIA due to impurity segregation during the growth process. Tuning the SIA by the delta-doping position, we can grow samples with almost equal degrees of structure and bulk inversion asymmetry. (c) 2009 American Institute of Physics. [DOI: 10.1063/1.3156027]
\end{abstract}

The generation, manipulation, and detection of spin polarized electrons in low dimensional semiconductors are at the heart of spintronics, see, e.g., Refs. 1-3. A versatile tool to achieve these goals provides spin-orbit coupling, which in quantum wells (QWs) based on III-V semiconductors removes the spin degeneracy of the energy bands. The spin splitting allows one to control the spin polarization by the electric field, determines the spin relaxation rate and can be utilized for all-electric spin injection. The lifting of the spin degeneracy is caused by spin-orbit interaction described by terms linear in electron wave vector $\boldsymbol{k}$ in the effective Hamiltonian $H_{\mathrm{SO}}=\Sigma \beta_{l m} \sigma_{l} k_{m}$, where $\boldsymbol{\beta}$ is a second rank pseudotensor and $\sigma_{l}$ is the Pauli matrix. The microscopic origin of these terms is the structure inversion and bulk inversion asymmetry (BIA), which lead to Rashba and Dresselhaus spin-orbit terms in $H_{\mathrm{SO}}$, respectively. ${ }^{1-3}$ The strength of the BIA spin splitting depends on the QW width, temperature, and electron density. In contrast structure inversion asymmetry (SIA) originates from the inversion asymmetry of the confining potential. The widely applied method to control SIA is the application of a gate voltage. Recently we demonstrated that in doped (110)-oriented GaAs QWs the proper choice of the $\delta$-doping layer position permits the growth of structures with controllable SIA. ${ }^{4}$

Here we investigate (001)-grown GaAs QWs and demonstrate that SIA is substantially affected by the segregation during growth of the structure. This is in contrast to (110)oriented QWs where doping segregation is suppressed. ${ }^{4}$ Our experiment explores the role of segregation and allow us to determine the growth conditions of structures with predetermined SIA. In this way we prepared the QWs with almost equal Rashba and Dresselhaus spin splittings. Such structures can be applied for the development of a nonballistic spin-field effect transistor, ${ }^{5}$ creation of a persistent spin helix ${ }^{6}$ and are characterized by a drastic increase in spin relaxation times. $^{7}$

The magnetogyrotropic photogalvanic effect (MPGE) is a photocurrent generation driven by the absorption of radiation in QWs in the presence of a magnetic field. The micro-

\footnotetext{
${ }^{\text {a) }}$ Present address: Solid State Physics Laboratory, ETH Zurich, Switzerland.

${ }^{b)}$ Electronic mail: sergey.ganichev@physik.uni-regensburg.de.
}

scopic origin of the phenomenon is explained in Refs. 3 and 8-10. Here in order to analyze the interplay between SIA and BIA, we apply the method based on the symmetry equivalence of the tensors describing the spin splitting and MPGE. ${ }^{4}$ From the phenomenological arguments the MPGE current density is given by ${ }^{10}$

$$
j_{\alpha}=\sum_{\beta \mu \nu} \phi_{\alpha \beta \mu \nu} B_{\beta}\left(E_{\mu} E_{\nu}^{*}+E_{\nu} E_{\mu}^{*}\right) / 2,
$$

where $\phi$ is a fourth rank pseudotensor being symmetric in the indices $\mu$ and $\nu, B_{\beta}$ are components of the magnetic field $\boldsymbol{B}$, and $E_{\mu}$ are the components of the radiation electric field $\boldsymbol{E}$. Excitation of (001)-grown QWs with unpolarized radiation at normal incidence in the presence of an external inplane magnetic field provides a straight forward method to obtain the relative strengths and signs of SIA and BIA. In this case Eq. (1) reduces to $j_{l}=\sum_{m} \gamma_{l m} B_{m}|\boldsymbol{E}|^{2}$, where $\boldsymbol{\gamma}$ is a second rank pseudotensor. Both MPGE and the spin splitting are characterized by the same anisotropy in space because they are described by equivalent second rank pseudotensors $\boldsymbol{\gamma}$ and $\boldsymbol{\beta}$ whose irreducible components differ by a scalar factor only.

In analogy to the band spin splitting the photocurrent can be decomposed into SIA and BIA contributions. In particular for an in-plane magnetic field applied along a cubic axis, e.g., $y$-direction, we obtain for longitudinal $\left(j_{y} \|[010]\right)$ and transverse photocurrents $\left(j_{x} \|[100]\right)$,

$$
j_{x}=\gamma^{\mathrm{SIA}} B_{y}|\boldsymbol{E}|^{2}, \quad j_{y}=\gamma^{\mathrm{BIA}} B_{y}|\boldsymbol{E}|^{2} .
$$

Here $\gamma^{\mathrm{SIA}}$ and $\gamma^{\mathrm{BIA}}$ are components of the tensor $\gamma$ due to SIA and BIA, respectively. Taking the ratio $j_{x} / j_{y}$ cancels the scalar factors, which contain all microscopic details and yields directly the ratio of SIA to BIA. Moreover, separate analysis of the photocurrents given by Eq. (2) reveals changes of the strength and the sign of SIA and BIA upon variation of external parameters.

In this experiment we investigated (001)-oriented Si- $\delta$-doped $n$-type $\mathrm{GaAs} / \mathrm{Al}_{0.3} \mathrm{Ga}_{0.7} \mathrm{As}$ structures grown by molecular-beam epitaxy at typical temperatures in excess of $600{ }^{\circ} \mathrm{C}$. Temperatures $T_{\delta}$ during the growth of the $\delta$-doping and subsequent layers, which determine the segregation, mobilities $\mu$, and carrier densities $n_{s}$, measured at room tem- 
TABLE I. Parameters of samples. Carrier densities $n_{s}$ (per QW-layer) and mobilities $\mu$ are room temperature values.

\begin{tabular}{ccccccc}
\hline \hline Sample & $\begin{array}{c}\text { Spacer } l \\
(\mathrm{~nm})\end{array}$ & $\begin{array}{c}\text { Spacer } r \\
(\mathrm{~nm})\end{array}$ & $\chi=l-r / l+r$ & $n_{s} 10^{11} 1 / \mathrm{cm}^{2}$ & $\mu 10^{3} \mathrm{~cm}^{2} / \mathrm{V} \mathrm{s}$ & $\begin{array}{c}T_{\delta} \\
\left({ }^{\circ} \mathrm{C}\right)\end{array}$ \\
\hline 1 & 20 & 165 & -0.78 & 1.2 & 7.2 & 615 \\
2 & 45 & 140 & -0.51 & 1.6 & 7.7 & 631 \\
3 & 70 & 115 & -0.24 & 1.4 & 8.0 & 631 \\
4 & 92.5 & 92.5 & 0 & 1.4 & 7.7 & 631 \\
$5 \mathrm{LT}$ & 92.5 & 92.5 & 0 & 1.0 & 7.9 & 490 \\
6 & 106.5 & 78.5 & 0.15 & 1.4 & 8.1 & 626 \\
7 & 111 & 74 & 0.20 & 1.4 & 8.1 & 632 \\
8 & 125 & 60 & 0.35 & 1.4 & 7.7 & 629 \\
9 & 140 & 45 & 0.51 & 1.4 & 7.9 & 630 \\
10 & 165 & 20 & 0.78 & 1.5 & 8.0 & 625 \\
\hline \hline
\end{tabular}

perature are given in Table I. Figure 1 sketches the conduction band edge of the multiple QW structure together with the corresponding $\delta$-doping position. All QWs have the same width of $15 \mathrm{~nm}$ but differ essentially in their doping profile. In all structures apart from sample 4 and 5LT, the doping layers are asymmetrically shifted off the barrier center either to the left or to the right. The impurities' Coulomb field yields an asymmetric potential profile inside the QWs. In order to describe the degree of asymmetry we introduce the parameter $\chi=(l-r) /(l+r)$, where $l$ and $r$ are the spacer layer thicknesses between QW and $\delta$-layers (Fig. 1 and Table I). Square shaped samples were investigated with edges of $5 \mathrm{~mm}$ length oriented along the [1 $1 \overline{0}]$ and [110] crystallographic axes. For photocurrent measurements ohmic contacts were alloyed on the sample corners and in the middle of each sample side allowing to probe the photocurrent along different directions, as displayed in Fig. 2. An external in-plane magnetic field $B= \pm 1 \mathrm{~T}$ is applied along either $x \|[100]$ or $y \|[010]$.

The MPGE is measured at room temperature by exciting the samples with unpolarized terahertz radiation under normal incidence, as sketched in the inset of Fig. 2(b). The pulsed radiation $(P \approx 5 \mathrm{~kW})$ is obtained applying an optically pumped $\mathrm{NH}_{3}$ molecular laser. ${ }^{9}$ The wavelength of $280 \mu \mathrm{m}$ was chosen to cause only free carrier absorption. Unpolarized light was obtained using a brass cone of $95 \mathrm{~mm}$ length with an angle of about $4^{\circ}$, which depolarizes the laser radiation as a result of multiple reflections.

Under unpolarized terahertz irradiation, we observed a photocurrent signal linearly increasing with rising magnetic field strength and changing the sign upon inversion of the magnetic field direction from $B_{+}>0$ to $B_{-}<0$. For convenience in the discussion below, we evaluate the data by calculation of $J=\left[J\left(B_{+}\right)-J\left(B_{-}\right)\right] / 2$, which yields solely the

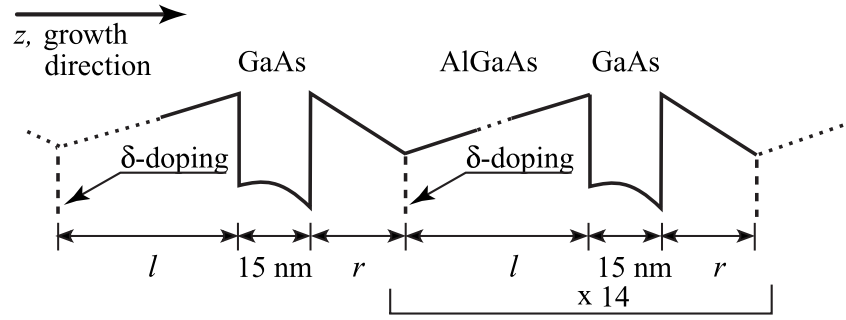

FIG. 1. Band profile (conduction band) of QWs indicating the doping position.
MPGE contribution. The signal is detected in both longitudinal and transverse directions with respect to the magnetic field orientation.

Figure 2(a) shows the ratio between $J_{x}$ and $J_{y}$ photocurrents as a function of the parameter $\chi$ obtained for magnetic fields of $\pm 1 \mathrm{~T}$ applied along the $y$-direction. As addressed above this quotient yields the ratio between SIA and BIA strengths. As an important result, Fig. 2(a) shows that the

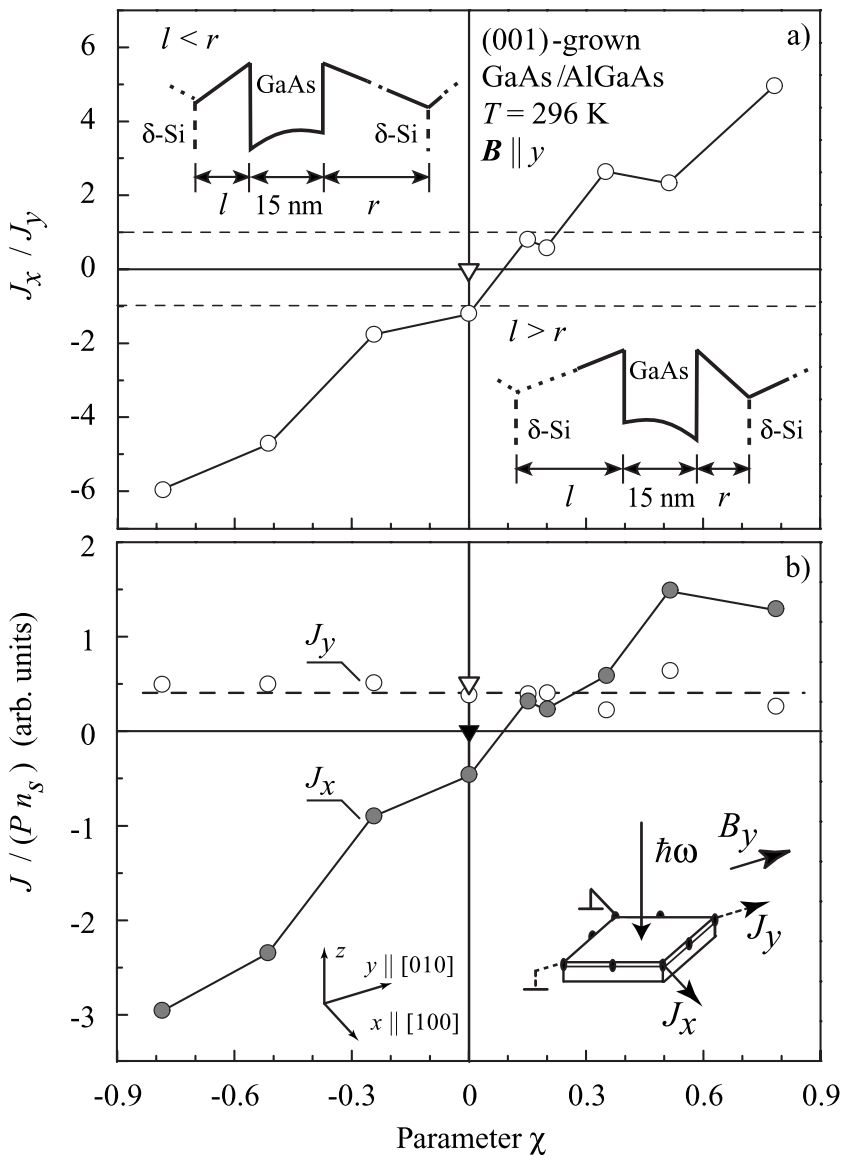

FIG. 2. (a) The ratio of the SIA and BIA contributions to the MPGE $J_{x} / J_{y}$ as a function of $\chi$. The triangle shows the result for sample 5LT grown at $T_{\delta}=490{ }^{\circ} \mathrm{C}$, the circles demonstrate the data for all other samples grown at $T_{\delta} \approx 630{ }^{\circ} \mathrm{C}$. Insets show the $\mathrm{QW}$ profile and the doping positions for $l<r$ and for $l>r$. (b) Dependence of $J / P n_{s}$ on the parameter $\chi$. The photocurrents are measured along and normal to $\boldsymbol{B} \| y$. Full and open symbols show $J_{x}$ and $J_{y}$, respectively (triangles are the data for sample 5LT). Inset: experimental geometry. 
SIA/BIA ratio strongly depends on the doping position and moreover, changes its sign for $\chi \approx 0.1$.

The dependence of both currents $J_{x}$ and $J_{y}$ on the parameter $\chi$ is shown in Fig. 2(b). Here we normalize the data by the free carrier concentration $n_{s}$ to enable a comparison of BIA and SIA in different samples. This is justified since for the Boltzmann distribution of carriers at room temperature the MPGE current is proportional to $n_{s}$ but independent of the electron mobility. ${ }^{10}$

Figure 2(b) demonstrates that while the longitudinal current is almost independent of $\chi$ the transverse current strongly depends on $\chi$. The results of Fig. 2(b) are in a full agreement with Eq. (2) demonstrating that the longitudinal current in this experimental geometry is solely due to BIA and the transverse current is caused by SIA only. In additional experiments we applied the magnetic field $\boldsymbol{B}$ along the $x$-direction. We observed that while the data on the transverse current remained the same the longitudinal photocurrent inverted its sign in all samples. This observation is also in agreement with Eq. (1), which yields for $\boldsymbol{B} \| x$ that the photocurrent is given by $j_{x}=-\gamma^{\mathrm{BIA}} B_{x}|\boldsymbol{E}|^{2}$ and $j_{y}=\gamma^{\mathrm{SIA}} B_{x}|\boldsymbol{E}|^{2}$.

The fact that the longitudinal current in both experimental geometries is independent of $\chi$ is expected for BIAinduced effects, which are obviously insensitive to the magnitude and the sign of $\chi$. The transverse current, in contrast, is caused by SIA and is very sensitive to the impurity potential. The variation of $\chi$ affects the degree of asymmetry and even changes the sign of the transverse photocurrent due to SIA for $\chi \approx 0.1$. Our results show that for $\chi<0.1$ the asymmetry is dominated by the potential of impurities placed on the left (substrate) side of the QW. Figure 2(b) demonstrates that in order to obtain a vanishing value of SIA (001)oriented samples must be asymmetrically doped $(l>r)$. This observation is attributed to the segregation of Si-impurities during molecular beam epitaxial growth and is in contrast to (110)-grown structures, where symmetrical doping results in a vanishing SIA. ${ }^{4}$ This essential difference is caused by the difference in growth conditions. Indeed, the growth temperature of high-quality (001)-oriented QWs is typically higher than $600{ }^{\circ} \mathrm{C}$, while (110)-structures are grown at $480{ }^{\circ} \mathrm{C} .{ }^{11}$ In order to suppress the segregation we prepared a symmetrically doped sample with reduced temperature during the $\delta$-doping $\left(T_{\delta}=490{ }^{\circ} \mathrm{C}\right)$. We find in this case that the MPGE current perpendicular to $\boldsymbol{B}$, which is caused solely by SIA, is almost equal to zero. This clearly demonstrates that the reduced growth temperature suppresses segregation and does not introduce additional SIA.

The next important observation is that for $\chi=0$ (sample 4) and $\chi \approx 0.17$ (samples 6 and 7) $\left|J_{x} / J_{y}\right| \approx 1$ [see Fig. 2(a)] indicating that SIA and BIA have equal strengths. In such samples the effects due to SIA and BIA cancel each other in either the $[1 \overline{1} 0]$ or $[110]$ crystallographic direction depend- ing on the relative sign of the SIA and BIA terms. ${ }^{7}$ For the transverse photocurrent caused by a magnetic field applied along one of $\langle 110\rangle$-axes the phenomenological theory yields $j_{\perp}=\left(\gamma^{\mathrm{SIA}} \pm \gamma^{\mathrm{BIA}}\right) B|\boldsymbol{E}|^{2}$, where the two signs correspond to the two magnetic field orientations. In particular for $\gamma^{\text {SIA }}$ $\approx \gamma^{\mathrm{BIA}}$ one can expect substantial differences in the transverse photocurrents for $\boldsymbol{B}$ applied along the [1 $\overline{1} 0]$ and [110] directions. Indeed, this anisotropy was observed: e.g., in sample 4 characterized by close to equal SIA and BIA strengths we found that the transverse signal changes its magnitude by a factor of six under rotation of an in-plane magnetic field $\boldsymbol{B}$ by $90^{\circ}$.

To conclude, we investigated SIA and BIA in (001)oriented GaAs/AlGaAs QWs at room temperature. The observed modulation of the SIA/BIA ratio by a shift of the doping position demonstrates that the impurity position plays an important role in the SIA. We verified that the high growth temperatures of high-quality (001)-oriented GaAs QWs add an additional factor to the SIA due to impurity segregation. We emphasize that our method to study SIA and BIA based on the MPGE can be applied even at room temperature, where many other methods cannot be used. Investigation of samples with different $\delta$-doping positions led to QWs with almost equal magnitudes of Rashba and Dresselhaus constants, which should exhibit extraordinary long spin relaxation times.

This work is supported by the DFG via programs (Contract Nos. SPP 1285 and SFB 689), RFBR, Russian President grant, and "Dynasty" foundation-ICFPM.

${ }^{1}$ R. Winkler, Handbook of Magnetism and Advanced Magnetic Materials (Wiley, New York, 2007).

${ }^{2}$ J. Fabian, A. Matos-Abiague, C. Ertler, P. Stano, and I. Žutić, Acta Phys. Slov. 57, 565 (2007).

${ }^{3}$ Spin Physics in Semiconductors, edited by M. I. D'yakonov (Springer, New York, 2008).

${ }^{4}$ V. V. Bel'kov, P. Olbrich, S. A. Tarasenko, D. Schuh, W. Wegscheider, T. Korn, C. Schüller, D. Weiss, W. Prettl, and S. D. Ganichev, Phys. Rev. Lett. 100, 176806 (2008).

${ }^{5}$ J. Schliemann, J. C. Egues, and D. Loss, Phys. Rev. Lett. 90, 146801 (2003).

${ }^{6}$ B. A. Bernevig, J. Orenstein, and S. C. Zhang, Phys. Rev. Lett. 97, 236601 (2006).

${ }^{7}$ N. S. Averkiev and L. E. Golub, Semicond. Sci. Technol. 23, 114002 (2008).

${ }^{8}$ V. V. Bel'kov and S. D. Ganichev, Semicond. Sci. Technol. 23, 114003 (2008).

${ }^{9}$ S. D. Ganichev and W. Prettl, Intense Terahertz Excitation of Semiconductors (Oxford University Press, Oxford, 2006).

${ }^{10}$ S. D. Ganichev, V. V. Bel'kov, S. A. Tarasenko, S. N. Danilov, S. Giglberger, C. Hoffmann, E. L. Ivchenko, D. Weiss, W. Wegscheider, C. Gerl, D. Schuh, J. Stahl, J. de Boek, G. Borghs, and W. Prettl, Nat. Phys. 2, 609 (2006).

${ }^{11}$ L. Pfeiffer, K. W. West, H. L. Stormer, J. P. Eisenstein, K. W. Baldwin, D. Gershoni, and J. Spector, Appl. Phys. Lett. 56, 1697 (1990). 